\title{
La retorica di Ulisse contro la pietas, da Dante a Tennyson
}

$\underline{\text { Abstract }}$

In antiquity, public speaking was based on a set of linguistic strategies for obtaining agreement, with a good command of rhetoric seen as an asset. In the post-classical period, rhetoric was vilified and its status diminished, due to the increasing (scientific) demand for tonal neutrality in discourse and the sublimation of oratory into printed material. In principle, Dante would have been respectful of the tradition governing epic speechmaking, but ultimately chose to depict Ulysses' fate in Inferno XXVI as a case of morally-suspect rhetoric, exaggerating the hero-sinner's deceptive skills and wanderlust as concepts antithetical to pietas, the idea of devotion espoused by Aeneas, Virgil's hero. Tennyson's depiction of Ulysses conforms in many ways to epic rhetorical conventions, though the postRomantic incarnation of the Homeric hero does not precisely dovetail with the figure of classical and medieval tradition, instead fitting the Victorian mould of a restless wanderer, more concerned with intellectual pursuits than social pietas and oratorical trickery. By undertaking a comparative analysis of the rhetorical devices and presentation of pietas in these two works, a distilled appreciation of the fluctuating significance of Ulysses and notions of duty from the medieval era to modern times can be achieved.

$\underline{\text { Keywords }}$

rhetoric; poetics; duty; medieval; Victorian

\section{L'arte della retorica e i doveri della pietas}

Nell'antica Roma, il parlare in pubblico era basato su un insieme di strategie linguistiche per ottenere un accordo. L'ars rhetorica era concepita come una metodologia per compiere un'orazione efficace e per comunicare razionalmente; ${ }^{1}$ John Bender e David Wellbery sottolineano in The Ends of Rhetoric che una buona padronanza delle tecniche oratorie 'served as a marker of authority and social standing' (Bender e Wellbery, 1990: 6). Tuttavia nel periodo post-classico, la retorica fu calunniata, e il prestigio ridotto da disciplina a collezione di tropi. Questo cambiamento fu senza dubbio inaugurato dalla necessità crescente (a causa della scienza) di una neutralità tonale nel discorso, e dalla sublimazione dell'oratoria nel materiale stampato ovunque in Europa dal sedicesimo secolo in poi. L'emergere del discorso liberale come lingua dello scambio ha perfino condotto Immanuel Kant a dichiarare (nella cinquantatreesima parte della Critique of the Power of Judgement) che la retorica era diventata simile a 'deceiving by a beautiful show (ars oratoria)' (Kant, 2000: 128). Le figure retoriche, soprattutto l'amplificatio e il captatio benevolentiae, sono tuttavia rimaste prevalenti nelle sfere letterarie e politiche fino all'era vittoriana (gli studiosi del latino e del greco, come Tennyson, erano particolarmente ben versati nel loro utilizzo), contro l'appello di John Locke in An Essay Concerning Human Understanding a liberare la lingua e il pensiero dalla 'rhetoric, that powerful instrument of error and deceit' (Locke, 1836: 373).

Dante, grande esperto di autori romani, soprattutto Virgilio ed Ovidio, era certamente conscio e, in principio, rispettoso della tradizione accademica (legata ai precetti aristotelici e ciceroniani) riguardo alla retorica epica, ma alla fine ha scelto di fare del destino di Ulisse una storia della retorica moralmente sospetta, esagerando le abilità ingannevoli dell'eroepeccatore come l'antitesi della pietas (la devozione alle divinità domestiche, alla famiglia ed ai vicini). Discepolo di Virgilio, Dante condivide l'idea del dovere esposta dall'eroe più celebre del poeta romano, Enea ('de' Romani il gentil seme' (Inferno XXVI.60)). I valori che dovevano essere la fondazione dell'ordine in tutto l'impero romano acquistano un'importanza chiave nella Commedia come soluzione al conflitto tra i Guelfi e i Ghibellini. Tali meriti, in particolare il rispetto per le responsabilità (soprattutto l'autorità di Dio e l'integrità della 
società), sono anche in accordo con i valori cristiani del poeta fiorentino. La retorica di Ulisse è raffigurata da Dante come un incitamento egoista e infido dei marinai ad intraprendere un viaggio che porta alla morte, con la narrazione dell'eroe-peccatore dimostrando chiaramente che ha trasgredito i limiti imposti da Dio, giacché le colonne di Ercole si presentano come marcatori della pietas in termini sia fisici che filosofici. ${ }^{2}$ Ulisse viene condannato per aver respinto la domesticità, e per esser passato in un mondo egocentrico:

né dolcezza di figlio, né la pieta

del vecchio padre, né 'l debito amore

lo qual dovea Penelopè far lieta,

vincer potero dentro a me l'ardore

ch'i' ebbi a divenir del mondo esperto

e de li vizi umani e del valore. (94-99)

Le allusioni nel discorso dell'eroe-peccatore, insieme al destino del viaggio alimentato retoricamente, contribuiscono teologicamente e moralmente alla realizzazione che l'orazion picciola di Ulisse merita la disapprovazione per l'opposizione della filosofia alla pietas; per il disprezzo dei principi sociali e divini.

Nonostante la fortuna fluttuante del mito di Ulisse e della retorica durante i secoli prima della visione di Tennyson dell'oratoria classica, anche il protagonista post-romantico è conforme in molti modi alle convenzioni retoriche, soprattutto nell'elocutio e nella perspicuitas del discorso. La versione di Ulisse dell'epoca vittoriana non combacia tuttavia con l'incarnazione della tradizione classica o medievale; anzi, possiede lo stampo del girovago inquieto, occupato dagli inseguimenti intellettuali. L'interpretazione tennysoniana di Ulisse appoggia solo a parole le nozioni della pietas familiare (che l'hanno richiamato a casa in Omero) attraverso l'inclusione di Penelope ('Match'd with an agèd wife' ('Ulysses', 3)) e di Telemaco ('This is my son' (35)) come figure unidimensionali, emblematici della responsabilità che l'eroe sta per abbandonare:

Most blameless is [Telemachus], centred in the sphere

Of common duties, decent not to fail

In offices of tenderness, and pay

Meet adoration to my household gods,

When I am gone. He works his work, I mine. (39-43)

L'incarnazione vittoriana di Ulisse è dunque molto più egocentrica del protagonista dantesco come seguace dell'indagine intellettuale ad ogni costo.

La dissezione di queste due opere deve pertanto avere uno scopo bipartito: in primo luogo, riguarda alla profondità della disapprovazione che attrae Ulisse a causa delle figure retoriche che utilizza; in secondo luogo, riguarda ai modi in cui la nozione della pietas è sovvertita come corollario della manipolazione oratoria.

\section{Dante, Inferno XXVI}

Come nell'epica classica, a metà del canto ventiseiesimo dell'Inferno comincia il discorso di Ulisse in medias res: 'Quando | mi dipartì da Circe' (90-91). Dante quasi raffigura il protagonista greco come l'autore di un epillio autobiografico, rendendo il viaggio verso la morte come la ricerca di un nuovo inizio nella tarda vita. La percezione romanzata di Ulisse come un avventuriero che vuole 'divenir del mondo esperto' (98) influisce indubbiamente sul trattamento dantesco delle riflessioni retoriche dell'eroe-peccatore, specialmente nell'enfasi sull'intelletto irrequieto e la volontà concentrata (due tratti messi in primo piano da Ovidio nel quattordicesimo libro delle Metamorfosi). La mente indagatrice di Ulisse (la controparte delle abilità retoriche secondo Cicerone) viene ben illustrata: 
l'ardore

ch'i' ebbi a divenir del mondo esperto

e de li vizi umani e del valore. (97-99)

Come rileva Giuseppe Mazzotta in Dante, Poet of the Desert, 'the primary concern of this canto is rhetoric', inquadrando l'impostazione dantesca di Ulisse 'within an ethical context' (Mazzotta, 1979: 71; 79). ${ }^{3}$ L'eroe-peccatore è conscio dei doveri verso Penelope e la famiglia, eppure è eroicamente costretto a seguire la chiamata misteriosa della possibilità di ottenere una consapevolezza maggiore di sé. Dante incanala nella propria visione di Ulisse la necessità umana di andare là dove nessuno è mai giunto prima: 'dov' Ercule segnò li suoi riguardi | acció che l'uom più oltre non si metta' (108-09). La litote dell'eroe-peccatore nella descrizione del suo tentativo di raggiungere il Monte Purgatorio senza la grazia divina è perfettamente adatta al combattente intellettuale di Omero:

quando n'apparve una montagna, bruna

per la distanza, e parvemi alta tanto

quanto veduta non avëa alcuna. (133-35)

La versione dantesca di Ulisse è quella di un uomo che ha puntato e perso tutto per aver cercato una coscienza superiore senza la grazia divina. L'idealizzazione dell'eroe-peccatore dell'inseguimento esperienziale, che va percepita come la follia eccessiva prodotta da una vita dedicata alla filosofia, ${ }^{4}$ potrebbe essere ispirata dall'esortazione di Sallustio nel primo verso della Bellum Catilinae che 'omnis homines, qui sese student praestare ceteris animalibus, summa ope niti decet, ne vitam silentio transeant veluti pecora [it becomes all men, who desire to excel other animals, to strive, to the utmost of their power, not to pass through life in obscurity, like the beasts of the field]' (Sallust, 2007: 27). Gli atti dell'eroe-peccatore l'hanno portato ad una crisi quasi esistenzialista, in cui la svalutazione della pietas (a causa dell'elevazione della conoscenza empirica al vertice della gerarchia devozionale) l'ha lasciato senza nessun riferimento con il quale possa misurare il tentativo arrogante di oltrepassare la mortalità, quindi Ulisse non si rende conto, o (peggio) sceglie di ignorare, i rischi insiti nel tentativo di acquisire la salvezza attraverso la fede assoluta nel potere della filosofia.

L'assenza sorprendente di interruzioni da parte di Dante-pellegrino durante i cinquantadue versi dell'esposizione di Ulisse suggerisce che il fiorentino è schiavo dell'aneddoto melodrammatico del navigatore invecchiato, e l'inferenza sottile della credenza classica che i sogni poco prima della veglia sono più significativi degli altri ('Ma se presso al mattin del ver si sogna' (7)) riporta rispetto per la tradizione classica. ${ }^{5}$ Ulisse, in comune con i grandi eroi tragici dell'antichità, utilizza efficacemente i precetti aristotelici della Retorica, soprattutto l'iperbole ('per cento milia | perigli' (112-13)), nello sviluppo dall'apostrofo ('O frati' (112)) in un captatio benevolentiae mellifluo basato sull'amplificatio (richiamando i discorsi di Enea ai suoi alleati nell'Eneide). ${ }^{6}$ In The Undivine Comedy, Teodolinda Barolini conferma che 'the narrative voice takes on more and more the note of dispassionate passion that will characterize its hero', manifestando una retorica che è 'austere, sublimely simple' (Barolini, 1992: 89). ${ }^{7}$ Gli enjambements prolungati (112-13; 114-16), le direzioni cosmiche ('di retro al sol' (117)), lo sviluppo dal genere giudiziario al deliberativo, combinati colla sequenza antitetica dell'iperbole e della litote, sostengono la grandiosità della ricerca. In seguito al partitio, c'è un cambio dal pathos alla logica nel peroratio, e il discorso si chiude con un aforismo quasi staziano: 'fatti non foste a viver come bruti | ma per seguir virtute e canoscenza' (119-20). Gli schemi sonori ricorrenti e le modulazioni allitterative creano un effetto incantatorio ed armonioso mentre Ulisse tesse l'incantesimo, promuovendo l'immagine di un guerriero veramente cerebrale. Michael Putnam propone che 'poetic language [...] used in strategic and surprising repetitions [...] subverts idealism' (Putnam, 1988: 173). Non va 
tuttavia dimenticato che Dante ha posto Ulisse nell'ottava bolgia per aver peccato contro $\mathrm{i}$ compagni come consigliere fraudolento, possibile grazie all'abuso della retorica dell'eroepeccatore, quindi ci deve essere un'equivalenza tra la disapprovazione dell'oratorio e la critica della moralità di Ulisse, nonostante l'ammirazione che il protagonista possa creare per la dedizione agli ideali filosofici. In Arduous Tasks, Lina Insana rileva che il peccato di Ulisse coinvolge 'both a misguided use of rhetoric and an inappropriate crossing of the boundaries that govern human learning' (Insana, 2009: 110), convalidando la giustizia (oratoria) del contrappasso ('ogne fiamma un peccatore invola' (42)), poiché tutto quello che rimane dell'inganno retorico di Ulisse è la lingua infida ('come fosse la lingua che parlasse' (89)).

La denigrazione dantesca della manipolazione oratoria è corroborata dalla metaforicità del nascondersi e del furto (distribuita come avviso nel preludio all'incontro tra Dante-pellegrino ed Ulisse) che va di pari passo con la punizione dell'eroe-peccatore. La caduta di Troia nel secondo libro dell'Eneide viene richiamata tramite 'l'agguato del caval' (59); Achille è adescato dal rifugio dove sua madre Deidamia l'aveva nascosto ('Deïdamìa ancor si duol d'Achille' (62)), evocando l'Achilleid di Stazio; Ulisse deve pagare 'del Palladio pena' (63), l'icona della sapienza che egli rubò dai troiani. Il ritratto dantesco dell'eroepeccatore con l'aspetto di un aristocratico spietato, intrigante e senza scrupoli (ma di talento) che applicò freddamente l'intelletto e notevoli talenti di eloquenza a fini sacrileghe fa parte della tradizione post-omerica, di cui faceva parte Virgilio. Anthony Cassell mette in primo piano le connotazioni negative del racconto di Ulisse: 'the main point of Dante's episode is that all of the actions attributed to Ulysses are doctrinally and generically typical of dissemblers [simulators, hypocritae]' (Cassell, 1981: 125). La versione dantesca di Ulisse conserva solo una consapevolezza superficiale della pietas (il concetto del 'debito amore' (95) allude ad Enea, il modello classico della devozione), e rifiuta infine il principio del dovere a Penelope e alla famiglia, scegliendo invece di seguire la chiamata misteriosa di un'autoconsapevolezza maggiore. L'amore ardente dell'avventura che spinge l'interpretazione dantesca di Ulisse ad ulteriori viaggi è un'abiura della pietas familiare. La presunzione schiacciante che esista un'equivalenza tra virtù e conoscenza viene sottolineata dal posizionamento dell'esperienza sensuale nella prima persona ('ma misi me per l'alto mare aperto' (100)) sopra la devozione familiare e teologica nella gerarchia di Ulisse dei doveri: 'né dolcezza di figlio, né la pieta $\mid$ del vecchio padre, né 'l debito amore' (94-95). Robert Harrison commenta che 'the world of language and the language of the world become in this sense the piety of the domestic, a piety Ulysses rejects as he passes beyond the last sign into the nameless' (Harrison, 1987: 1048). Dante riconosce attentamente di non esser disposto a salire a tali altezze ('lo 'ngegno affreno [...] | perché non corra che virtù nol guidi' (21-22)), e la conclusione del racconto di Ulisse è inequivocabilmente disastrosa:

Tre volte il fé girar con tutte l'acque a la quarta levar la poppa in suso e la prora ire in giù com' altrui piacque infin che 'l mar fu sovra noi richiuso. (139-42)

Tutto è perso, a parte Dio che presiede sull'oceano immenso e silenzioso in un finale calmo, valutato da John Freccero nel contributo a The Cambridge Companion to Dante come 'a Christian critique of philosophical presumption' (Freccero, 1993: 174). ${ }^{8}$ L'ultimo verso del canto richiama un inquietante antecedente virgiliano (dell'unica nave della flottiglia troiana distrutta in una tempesta), ${ }^{9}$ inoltre al destino terribile dell'indovino Anfiarao nella Tebaide di Stazio. ${ }^{10}$ Questi echi sono certamente significativi per l'aspirante eroe greco, evocando una selezione sconsolante di figure pagane che servono come modelli per il viaggio avventato e la scomparsa infernale di Ulisse. 
Come l'implicazione allegorica del viaggio omerico di Ulisse è il trionfo dell'uomo che raggiunge uno stato superiore di illuminazione dopo aver superato sfide innumerevoli ed i propri demoni, così nella Commedia il Monte Purgatorio ha una connotazione simile. Ulisse è comunque pagano ed immeritevole, quindi è censurato principalmente per aver tentato di raggiungere uno stato superiore della comprensione (il Monte Purgatorio) senza la grazia divina (oltre all'ambizione ed all'invenzione di inganni). Come nota Umberto Bosco in Dante, le colonne di Ercole, oltre le quali Ulisse osa procedere, rappresentano i limiti 'posti da Dio per una ragione supremamente importante' (Bosco, 1967: 190), il confine che nessun mortale può trasgredire senza subire le conseguenze di tale empietà. In questa luce, il rapporto tra l'eroe-peccatore dantesco ed Enea espone un'ironia prodigiosa, poiché Ulisse tenta di competere con la pietas del troiano. La subordinazione di Ulisse degli eventi dell'Eneide in un esercizio di addomesticamento ('presso a Gaeta, | prima che sì Enëa la nomasse' (92-93)) fornisce ulteriori munizioni per condannare l'eroe-peccatore, e David Thompson afferma che 'Ulysses appears as the antitype of Aeneas' (Thompson, 1967: 46). Ulisse rinuncia ai valori familiari inerenti al battesimo dell'isola in omaggio alla bambinaia di Enea perché l'eroepeccatore non è affatto interessato né al nominare né all'appropriarsi delle terre a causa della 'chiamata maggiore' della ricerca filosofica. Ulisse asserisce una superiorità (falsa) sopra il fondatore di Lavinium perché Enea non ha nessun interesse a 'l'alto mare aperto' (100) ed è conforme alle gerarchie tradizionali del comportamento. Dante termina un racconto pagano con un approccio cristiano, evocando Ulisse come figura nella ricerca della conoscenza amplificata senza la grazia divina, di modo che l'eroe-peccatore incarna il conflitto tra mente e anima, tra ragione e fede. Può darsi che Dante senta un certo rispetto per la filosofia di Ulisse, ma il fiorentino non può perdonare la presunzione che ha causato il tormento eterno. L'eccessivo orgoglio intellettuale di Ulisse lo ha portato ad oltrepassare i confini di Dio in un 'folle volo' (125), e il destino sinistro dell'eroe-peccatore è un avvertimento a chiunque tenti di salvarsi attraverso la ragione.

\section{Tennyson, 'Ulysses'}

A differenza del racconto dantesco in cui il protagonista relativamente giovane persegue un'odissea alternativa, la storia di Tennyson si centra sull'eroe in finis res. La visione di Ulisse del poeta vittoriano differisce dal ritratto della Commedia principalmente a causa dell'atteggiarsi post-romantico (ed elegiaco), in particolare nella sezione giudiziaria:

all times I have enjoyed

Greatly, have suffered greatly, both with those

That loved me, and alone. (7-9)

La giustapposizione avverbiale del godimento iperbolico alla sofferenza rafforza il valore dell'ego, con il 'hungry heart' (12) di Ulisse suggerendo una visione post-illuminista delle ricompense dell'esperienza. La sete (scientifica) della conoscenza empirica definisce l'eroeviandante, come propone Clyde Ryals in Theme and Symbol in Tennyson's Poems to 1850: 'Ulysses is not the hero of a classical past, but, instead, the perplexed, restless wanderer of Victorian England' (Ryals, 1964: 126). Il protagonista tennysoniano è inoltre molto più fissato della versione dantesca sulla reputazione, enumerando un res gestae alla maniera di un monarca anziano, e cercando di abbandonare i doveri della pietas. Gli atti di Ulisse sono riassunti nell'ellissi fattuale di 'Much have I seen and known; cities of men | And manners, climates, councils, governments' (13-14). Una grande parte del discorso che precede la menzione di Telemaco evoca la tradizione classica, ${ }^{11}$ con una conclusione malinconica:

$$
\text { yearning in desire }
$$

To follow knowledge like a sinking star, Beyond the utmost bound of human thought. (30-31) 
L'iperbole cosmica dell'ultimo verso di questa similitudine, che comporta qualche sfumatura dantesca ('di retro al sol, del mondo sanza gente' (Inferno XXVI.117)), disprezza le funzioni quotidiane di un re, di un marito e di un padre. Ulisse preferisce lasciarsi alle spalle tutte le trappole mortali per arrivare ad un mondo dove non c'è nessun obbligo del genere (un'indicazione della mentalità egoista ed anti-pietas del protagonista tennysoniano). Elias Chiasson osserva nel contributo a Critical Essays on the Poetry of Tennyson che 'Tennyson, whose affinities are Virgilian and Dantean rather than Homeric, recognises that Ulyssean determination and courage, necessary as they are at certain junctures, are to be valued only if they contribute to the good life, personal and social' (Chiasson, 1960: 172). Il prolegomeno di Ulisse stabilisce un potente manifesto del genere deliberativo:

It little profits that an idle king, By this still hearth, among these barren crags,

Match'd with an agèd wife, I mete and dole

Unequal laws unto a savage race,

That hoard, and sleep, and feed, and know not me. (1-5)

L'individuo viene brutalmente separato dalla pietas comune e cerca la soddisfazione nell'inseguimento egoista della conoscenza elevata. Il tono di Ulisse è filippico ('I mete and dole' (3)), con aggettivi frivolamente dispregiativi ('barren' (2); 'agèd' (3); 'savage' (4)) prefigurando il disprezzo dell'ordine, dell'unità e dell'amore che viene rilevato nei confronti di Telemaco. L'insensibilità di Ulisse verso Penelope sovverte la pietas uxoria in modo straordinariamente simile all'affermazione del protagonista dantesco ('né 'l debito amore | lo qual dovea Penelopè far lieta | vincer potero dentro a me l'ardore' (Inferno XXVI.95-97)). Può darsi che tale amarezza nasca dalla realizzazione di Ulisse che la vita cercata per tanto tempo e recuperata con grande forza lo spinge a 'rust unburnished, not to shine in use' (23). L'eroe ha perso lo scopo e la vita (ad)domestica(ta) è definita dalla stasi, da quello che Ward Hellstrom sintetizza in On the Poems of Tennyson come una 'absence of action, fruition, change, purpose, and progress that are associated with life' (Hellstrom, 1972: 19). Il desiderio di illimitatezza in 'I cannot rest from travel: I will drink | Life to the lees' (6-7) è indicativo della voglia di Ulisse di capire il mondo attraverso il ragionamento e l'esperienza, oltre al dispregio per la pietas sociale imposta su di lui. In nessun luogo è il disprezzo di Ulisse dei doveri (e il talento per la retorica ostentata) dimostrato meglio che nell'ipotiposi dell'obiettivo della ricerca vaga (evocando allo stesso tempo la delusione della vita):

all experience is an arch wherethrough

Gleams that untravelled world, whose margin fades

For ever and for ever when I move. (19-21)

Sia il cambiamento del ritmo sia l'ampliamento della prospettiva in questi versi sconvolgono magistralmente i tratti (capricciosamente) epici delle reminiscenze precedenti. Tutta l'esperienza iliadica dell'eroe impallidisce in confronto alla gravità improvvisa dello spostamento prospettico da una visione grandangolare del passato ad un primo-piano estremo sul futuro, con il suo fascino dell'ignoto.

Il passaggio più denso retoricamente sorge quando l'eroe si trova a confrontare l'enigma del figlio. Il tono paternalistico ed importuno ('To whom I leave the sceptre and the isle' (34)) trasmette (poco sottilmente) il disprezzo per Telemaco. Ryals rileva che l'unico discendente di Ulisse rimane 'almost completely colourless' (Ryals, 1964: 128) durante tutto il discorso del padre:

by slow prudence to make mild

A rugged people, and through soft degrees 
Subdue them to the useful and the good.

Most blameless is he. (36-39)

La mancanza di interiezione del figlio pone l'accento sul divario tra le generazioni, e la paralessi ambivalente di Ulisse verso le capacità (e la potenzialità) dell'erede in un genere negativamente epidittico ('slow prudence' (36); 'soft degrees' (37); 'Most blameless' (39)) fa apparire l'uomo anziano come se stesse parodiando le tradizioni della saggezza politica dell'antichità. A tutti gli effetti, Ulisse può a mala pena attendere di abbandonare le responsabilità che gli impediscono di affrontare la fame intellettuale. James Kincaid dichiara in Tennyson's Major Poems che un 'sense of casual superiority is carried largely by his diction, which is weary, cliché-filled, "official" language' (Kincaid, 1975: 44). La sincrisi è resa esplicita ('He works his work, I mine.' (40)) appena prima che l'eroe faccia un gesto plateale tetico nello scioglimento, chiedendo ai marinai di fare un ultimo viaggio verso l'ignoto in una dichiarazione assoluta di arroganza vittoriana (la gratificazione dell'ego è paragonata alla pietas sociale di Telemaco): 'my purpose holds | To sail beyond the sunset' (59-60). La falsità retorica molto abile del captatio benevolentiae (('My mariners, | Souls that have toiled, and wrought, and thought with me' (45-46)) non può nascondere l'indifferenza di Ulisse al destino degli uomini ('It may be that the gulfs will wash us down' (62)), e la serenità del tono pone l'accento sul sacrificio egoista di molti animi alla ricerca dell'obiettivo filosofico. Kincaid osserva che 'Ulysses seems to insist absolutely on the final separation of the individual from communal values' (Kincaid, 1975: 43). Può darsi che l'eroe-peccatore dantesco sia incurante dei compagni allo stesso modo, ma la sua retorica è molto superiore a quella del protagonista tennysoniano, perché la prima versione di Ulisse fa appello alle sensibilità elevate dei compagni in un'ipotiposi che li incita attraverso un appello (fraudolento) ad una curiosità condivisa ('fatti non foste a viver come bruti $\mid$ ma per seguir virtute e canoscenza' (Inferno XXVI.119-20)). L'eroe-viandante di Tennyson è più distaccato ed evoca un senso gioviale della superiorità sardonica, sottolineato dal possessivo nella prima persona singolare: 'my purpose' (59). La dialettica dietro il ragionamento diventa chiara: Ulisse può restare a casa ed appassire, oppure scegliere l'orizzonte infinito del girovago romantico e partire per un viaggio. Ryals stabilisce che il tragitto scelto da Ulisse 'is not stimulated by wisdom, [and] will result in his abnegating his duties as king, husband, and father' (Ryals, 1964: 180). Il protagonista vittoriano crea una gerarchia retorica in cui la curiosità intellettuale è elevata (attraverso il linguaggio iperbolico) sopra la pietas della sfera domestica. La natura impervia dell'eroe-viandante è rilevata nei duri monosillabi (pleonastici) con cui fa riferimento al suo popolo: 'That hoard, and sleep, and feed, and know not me' (5). La spavalderia del protagonista vittoriano viene canalizzata nel disprezzo delle funzioni fondamentali dell'umanità. Le sue esperienze l'hanno reso così diverso del suo popolo che Ulisse non può più capire le loro preoccupazioni, quindi sceglie di rimanere libero e di perseguire le proprie ideali intellettuali e 'nobili'.

I viaggi omerici iniziano spesso al crepuscolo, quindi qualche ammirazione sottile per la tradizione classica è evocata nel ritratto tennysoniano di Ulisse partendo per un ultimo viaggio nella sera della vita. Un senso di movimento deliberatamente misurato è creato dalla dominanza del fonema [o] allungato e dal cambiamento dei livelli immaginosi, in combinazione con un appello ai sensi più immediati (la vista e l'udito) in un'altra ipotiposi:

The lights begin to twinkle from the rocks:

The long day wanes: the slow moon climbs: the deep

Moans round with many voices. (54-56)

Viene così richiamato un senso cosmico dello scopo di Ulisse immediatamente prima del passaggio ad un genere positivamente epidittico nell'apostrofo dei marinai. La peroratio 
combina la curiosità essenziale col coraggio temerario, in comune coi marinai leggendari dell'epoca vittoriana (pure col protagonista dantesco). William Robson suggerisce nel contributo a Critical Essays on the Poetry of Tennyson che i due racconti non sono tuttavia presentati nello stesso modo: 'Dante's character, whatever else he may be, is certainly in the first place a seaman telling a yarn; Tennyson's speaker is a self-conscious poet' (Robson, 1960: 158). In contrasto con Dante, Tennyson non è particolarmente sprezzante della retorica di Ulisse in sé. Il senso della manipolazione oratoria di terze parti è alquanto diminuito, soprattutto poiché la partitio di Ulisse è notevolmente vaga, e la ripetizione di 'It may be' (62; $63)$ evoca il senso di un tricolon incompleto. Può darsi che il poeta vittoriano abbia più simpatia per l'eroe a causa della giustapposizione (antitetica?) di una volontà indomabile con un corpo invecchiato? La litote eroica al termine del discorso di Ulisse è un corollario della lingua esaltata con cui esprime il bisogno umano di andare oltre i confini della conoscenza:

Though much is taken, much abides; and though

We are not now that strength which in old days

Moved earth and heaven; that which we are, we are;

One equal temper of heroic hearts,

Made weak by time and fate, but strong in will

To strive, to seek, to find, and not to yield. (65-70)

C'è un notevole senso di serena fiducia (in particolare nella geminazione del sessantasettesimo verso) in questa proposta di sacrificare qualche vita umana alla ricerca di un'esperienza trascendentale (che trionfo dell'ambizione scientifica dell'illuminismo nei confronti della conoscenza empirica del mondo!). Questo desiderio di arrivare ad un 'mondo sanza gente' (Inferno XXVI.117) è in comune col protagonista dantesco. Tale ambizione attesta una delle differenze più notevoli dalla tradizione omerica che condividono entrambi i poeti, nella misura in cui è l'assenza della società che Ulisse sta cercando, piuttosto della pietas che comporta la cura dei vicini. In The Aesthetic of the Victorian Dramatic Monologue, Megan Painter riconosce che 'Tennyson's version is a mixture of Dantesque desire for knowledge and the reckless courage of the English sea legends' (Painter, 2000: 4). Ė possibile che il protagonista tennysoniano sia temerario, ma è anche costretto dall'impulso più forte (rispetto alle esigenze della pietas) di abbandonare il mondo conosciuto ed andare oltre le colonne di Ercole, puntando a 'touch the Happy Isles' (63), dove la sua fede in una gerarchia al di fuori della responsabilità sociale verrà ricompensata in un (quasi) Eliseo oltre i limiti del dovere e del moralismo; dove sarà riunito con Achille, che scelse una vita breve e gloriosa, antitetica alla pietas (come Ulisse avrebbe preferito). Tennyson può essere immaginato come meno critico della retorica di Ulisse rispetto a Dante. L'eroe-viandante non è eccessivamente vilipeso per gli abusi della retorica, ma piuttosto per il rifiuto della pietas sociale.

\section{Il dovere comune e la trasgressione retorica, dal medioevo all'epoca moderna}

Dante e Tennyson condividono un senso di correttezza classica per quanto riguarda l'antico esponente dell'astuzia nei loro racconti, ma il desiderio di Ulisse di trovare un mondo al di fuori dei limiti mortali è un'inversione degli ideali cristiani e vittoriani. Di conseguenza, la nave del protagonista dantesco viene affondata da Dio, mentre la fine del racconto tennysoniano potrebbe presagire un destino simile per l'incarnazione moderna. Sono le due versioni di Ulisse giudicate nello stesso modo? Il ventiseiesimo canto dell'Inferno rivela un doppio motivo della dannazione: in primo luogo, l'eroe-peccatore rifiuta la pietas, portando egoisticamente i compagni lontano da un mondo del dovere e della devozione in un viaggio che finisce nella morte di tutti; in secondo luogo, il protagonista dantesco impiega stratagemmi retorici per raggiungere quest'obiettivo. Tennyson, nonostante qualche affinità virgiliana condivisa con Dante, rimprovera ad Ulisse solo il rifiuto della pietas sociale, 
piuttosto che qualche manipolazione oratoria in particolare. La mancanza della fede nelle strutture comuni e nella coesione è quello che, per il poeta vittoriano, svaluta l'obiettivo filosofico di Ulisse, in contrasto alla condanna teologico-retorica di Dante. La divergenza di atteggiamento tra Dante e Tennyson riguarda strettamente il ruolo diverso della retorica nelle due società. I due poeti evocano il disprezzo per l'abbandono di Ulisse di coloro cui doveva devozione perché il valore della pietas (anche se alquanto trasformata) resta elevato in entrambi i periodi, però la retorica dell'eroe non è pericolosa per la società di Tennyson tanto quanto Dante la giudica, quindi la censura nel testo vittoriano di Ulisse è diminuita.

Per capire meglio la condanna dantesca di Ulisse, è fruttuoso considerare più attentamene il ruolo importante che la retorica giocava nella cultura medievale. In Classical Rhetoric and its Christian and Secular Tradition, George Kennedy evidenzia che la 'rhetoric was part of basic education' (Kennedy, 1980: 180). Non va dimenticata l'importanza delle arti oratorie nella vita sociale del medioevo, soprattutto perché la capacità di persuasione legata alla retorica era intrinseca al potere temporale ed alla teologia, cioè i due poli di riferimento per la gente. L'epoca in cui Dante scriveva era pervasa dai concetti classici, e per gli intellettuali era molto importante saper schierarsi con i modelli dell'antichità. Questo impegno diede adito alla rielaborazione notata da Henry Taylor in The Classical Heritage of the Middle Ages: 'conscious imitation of the Roman past prevailed' (Taylor, 1957: 233). Per la società medievale, la retorica aveva lo stesso valore elevato dell'era romana, perciò qualsiasi abuso andava duramente condannato a causa dell'impatto che possa avere sulla vita comune. Evelyn Vitz indica in Medieval Narrative and Modern Narratology che gli autori medievali creavano una letteratura 'not of expression, but of impression' (Vitz, 1989: 33). L'uso corretto della retorica era obbligatorio, per paura che il suo abuso destabilizzi la coerenza ed i valori del mondo, quindi un personaggio come Ulisse che manipola le persone di cui è responsabile va punito doppiamente, sia per l'uso improprio delle arti oratorie che per la fede nell'empirismo al posto della religione.

Nell'epoca vittoriana, la forza della retorica era diventata così svalutata che i discorsi oratori non minacciavano la stabilità della società nello stesso modo. Come rileva Robert Ellison in The Victorian Pulpit, il periodo in cui Tennyson scriveva vide 'the culmination of a shift from an ornamental oral rhetoric to a plainer, more natural, more literary approach' (Ellison, 1998: 28). La retorica epica dell'antichità non era più adatta alle circostanze della vita comune: pochi credevano alle grandi arti oratorie di un personaggio come Ulisse. Diarmid Finnegan in una discussione della retorica nell'epoca vittoriana sottolinea le 'changing norms of public speech' (Finnegan, 2011: 64). L'idea che il parlare in pubblico al tempo di Tennyson aveva assunto valori diversi (di fronte ad un pubblico concentrato sul contenuto al posto della dizione) viene potenziata da Elisabeth Hansot nell'analisi del legame tra la retorica moderna ed i doveri civili: 'to judge content, not delivery, suggests the new values of transparency and neutrality in speech' (Hansot, 2004: 37). La retorica del protagonista tennysoniano allora non minaccia la società come quella della versione dantesca, e la fine della storia vittoriana è pregna di una tristezza pratica, invece di una condanna teologico-retorica.

\section{Bibliografia}

Alighieri, D. 1994 [1321]. La Commedia, a cura di G. Petrocchi, vol. 2. Firenze: Le Lettere.

Barolini, T. 1992. The Undivine Comedy: Detheologizing Dante. Princeton, NJ: Princeton University Press.

Bender, J. \& Wellbery, D. E. 1990. The Ends of Rhetoric: History, Theory, Practice. Stanford, CA: Stanford University Press.

Bosco, U. 1967. Dante: 'L'Inferno'. Torino: ERI.

Cassell, A. K. 1981. The Lesson of Ulysses. Dante Studies, 99: 113-31. 
Chiasson, E. J. 1960. Tennyson's 'Ulysses': A Re-interpretation, In: Critical Essays on the Poetry of Tennyson, a cura di J. Killham. Londra: Routledge \& Paul, pp. 164-73.

Ellison, R. H. 1998. The Victorian Pulpit: Spoken and Written Sermons in Nineteenth-Century Britain. Selinsgrove, PA: Susquehanna University Press.

Finnegan, D. A. 2011. Exeter-Hall Science and Evangelical Rhetoric in Mid-Victorian Britain. Journal of Victorian Culture, 16: 46-64.

Freccero, J. 1993. Introduction to Inferno. In: The Cambridge Companion to Dante, a cura di R. Jacoff. Cambridge: Cambridge University Press, pp. 172-91.

Hansot, E. 2004. Hearing Voices: Rhetoric, Imitation, and Civic Competence. Journal of Education, $185(2)$ : $27-$ 46.

Harrison, R. P. 1987. Comedy and Modernity: Dante's Hell. Modern Language Notes, 102(5): $1043-61$.

Hellstrom, W. 1972. On the Poems of Tennyson. Gainesville, FL: University of Florida Press.

Homer. 1866 [705 BCE]. Odyssey, a cura di H. Hayman. Londra: David Nutt.

Horace. 1998 [23 BCE]. Epodes and Odes, a cura di D. H. Garrison. Norman, OK: University of Oklahoma Press.

Insana, L. 2009. Arduous Tasks: Primo Levi, Translation, and the Transmission of Holocaust Testimony. Toronto, ON: University of Toronto Press.

Kant, I. 2000 [1790]. Critique of the Power of Judgement, trad. da P. Guyer \& E. Matthews. Cambridge: Cambridge University Press.

Kennedy, G. 1980. Classical Rhetoric and its Christian and Secular Tradition from Ancient to Modern Times. Chapel Hill, NC: University of North Carolina Press.

Kincaid, J. R. 1975. Tennyson's Major Poems: The Comic and Ironic Patterns. New Haven, CT: Yale University Press.

Locke, J. 1836. An Essay Concerning Human Understanding. Londra: T. Tegg \& Son.

Mazzotta, G. 1979. Dante, Poet of the Desert: History and Allegory in the 'Divine Comedy'. Princeton, NJ: Princeton University Press.

Ovid. 1996 [20 BCE]. Heroides XVI-XXI, a cura di E. J. Kenney. Cambridge: Cambridge University Press.

Painter, M. G. 2000. The Aesthetic of the Victorian Dramatic Monologue. Lewiston, NY: Edwin Mellen Press.

Putnam, M. C. J. 1988. Virgil's Inferno. Materiali e discussioni per l'analisi dei testi classici, 20-21: 165-202.

Robson, W. W. 1960. The Dilemma of Tennyson. In: Critical Essays on the Poetry of Tennyson, a cura di J. Killham. Londra: Routledge \& Paul, pp. 155-63.

Ryals, C. de L. 1964. Theme and Symbol in Tennyson's Poems to 1850. Philadelphia, PA: University of Pennsylvania Press.

Sallust. 2007 [40 BCE]. Bellum Catilinae, a cura di J. T. Ramsey. Oxford: Oxford University Press.

Statius. 2003 [92 CE]. Thebaid I-VII, a cura di D. R. Shackleton-Bailey. Cambridge, MA: Harvard University Press.

Taylor, H. 1957. The Classical Heritage of the Middle Ages. New York, NY: Frederick Ungar.

Tennyson, A. 2009 [1842]. The Major Works, a cura di A. Roberts. Oxford: Oxford University Press.

Thompson, D. 1967. Dante's Ulysses and the Allegorical Journey. Dante Studies, 85: 33-58.

Virgil. 1868 [19 BCE]. Aeneid, a cura di T. Chase. Philadelphia, PA: Eldredge \& Brother.

Vitz, E. B. 1989. Medieval Narrative and Modern Narratology. New York, NY: New York University Press.

\footnotetext{
${ }^{1}$ Alla base, la retorica coinvolse tre generi principali: il giudiziario, il deliberativo e l'epidittico. Il genere giudiziario era per i tribunali e per fare discorsi su eventi passati; il genere deliberativo era per la politica, per
} 
dare consigli o per persuadere qualcuno verso una decisione controversa; il genere epidittico era adatto all'elogio od alla denigrazione di una terza parte (occasionalmente non presente; spesso morta).

${ }^{2}$ Come limiti imposti da Dio, le colonne rappresentano il confine del mondo cui i mortali devono accontentarsi. Ulisse scarta i propri doveri devozionali per cercare un'esistenza non legata ai vincoli familiari, sociali e religiosi.

${ }^{3}$ Il trattamento complesso della retorica viene riconosciuto da Mazzotta come 'a creative discipline that fashions history' (Mazzotta, 1979: 73) per tutta la cantica, quindi la storia di Ulisse va compresa non solo come una riscrittura della mitologia antica, ma anche come un episodio importante nella valutazione dantesca delle arti oratorie in tutta la Commedia.

${ }^{4}$ Ulisse aderisce ad una visione egocentrica di un piano esistenziale sopra la responsabilità teologica e sociale a causa della sua fede infallibile nel potere della ragione di raggiungere l'illuminazione, così che la devozione a nozioni non derivanti all'interno di sé diventa inutile. L'unica autorità alla quale l'eroe-peccatore risponde è il proprio intelletto.

${ }^{5}$ Seguendo Ovidio, Eroidi XIX.195-96: 'namque sub aurora iam dormitante lucerna | somnia quo cerni tempore vera solent [For, just before dawn, as my lamp was already dying, $\mid$ At the time when dreams are said to be true]' (Ovid, 1995: 66). Aurora, dea dell'alba, doveva concedere la comprensione mentre illuminava il mondo.

${ }^{6}$ Virgilio, Eneide I.198-203: 'O socii, neque enim ignari sumus ante malorum | [...] revocate animos, maestumque timorem $\mid$ mittite [O, my companions, we have not been ignorant before now of calamities $\mid[\ldots]$ Lift up your spirits, banish your fear!]' (Virgil, 1868: 14). C'è anche una risonanza con l'evocazione oraziana di Teucro negli Odi (I.vii.25-26), che cerca di convincere i compagni a fare un viaggio analogamente fatale: 'Quo nos cumque feret melior fortuna parente | ibimus, o socii comitesque [Wherever Fortune, better than that of my father, may bear us, | We will go, o companions and friends]' (Horace, 1998: 35).

${ }^{7}$ Barolini rileva che ci sono state diverse 'extreme readings' (Barolini, 1992: 50) della visione dantesca di Ulisse. Un gruppo trova nel canto una simpatia poetica per la ricerca di Ulisse (e la retorica che la giustifica), mentre l'altro vede un moralismo autorale che rifiuta gli obiettivi intellettuali di Ulisse. Tali interpretazioni 'rob the episode of its tension and deflate it of its energy' (Barolini, 1992: 50), poiché il primo non riconosce che Dante colloca Ulisse nell'ottava bolgia dell'Inferno, mentre il secondo minimizza il valore elevato dell'eroe-peccatore rispetto agli altri peccatori. Considerando il 'textual and poetic privileging' (Barolini, 1992: 51) che implicano le molteplici riapparizioni di Ulisse in ogni cantica (viene nominato sia in Purgatorio XIX che in Paradiso XXVII), non si deve tralasciare la convergenza di Dante ed Ulisse al livello dell'ambizione intellettuale. La retorica dell'eroe-peccatore può essere anche capita come un tentativo dantesco di giustificare il ruolo di scriba Dei.

${ }^{8}$ Freccero nota che 'the self-reliance of Ulysses was interpreted by Dante [...] as a form of presumption of which the young Dante - especially the Dante of the Convivio - might himself have been guilty' (Freccero, 1993: 174). Si può capire la fine infernale dell'ambizione intellettuale e dell'autosufficienza di Ulisse come una critica dantesca del proprio orgoglio giovanile. Termina male la supponenza di realizzare un progetto fuori norma, e la retorica di Ulisse viene castigata perché spinge gli ascoltatori manipolati ad oltrepassare ciò che è prescritto da un potere più alto.

${ }^{9}$ Virgilio, Eneide I.116-17: 'at illam ter fluctus ibidem | torquet agens circum, et rapidus vorat aequore vortex [But three times the circling flood | swiftly spun the bark, and it was devoured by the engulfing seas]' (Virgil, 1868: 12). La nave che trasporta i liciani affonda in vista della terra (e della sicurezza), come fa quella di Ulisse. Sono tutti e due navi dei dannati.

${ }^{10}$ Stazio, Tebaide VII.821-23: 'respexitque cadens caelum, campumque coire | ingemuit, donec levior distantia rursus $\mid$ miscuit arva tremor lucemque exclusit Averno [Sinking, he looked back at the heavens, and groaned as the plain met above him, | until a fainter shock joined once more | the parted fields, and shut out the daylight from Avernus]' (Statius, 2003: 458). Anfiarao cade in un baratro nel suolo, poi la terra chiude sopra di lui. È condannato ad un destino orribile, tutto come Ulisse ed il suo equipaggio.

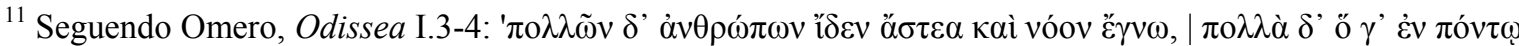

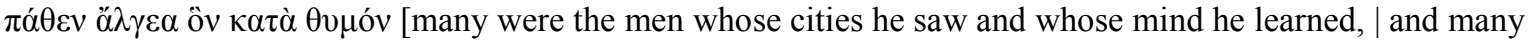
the woes he suffered in his heart upon the sea]' (Homer, 1866: 3-4). 